\title{
Giorgio Chiosso, Libri di scuola e mercato editorial. Dal primo Ottocento alla Riforma Gentile
}

Milano, Franco Angeli, 2013, 223 p.

\section{Michel Ostenc}

\section{(2) OpenEdition}

\section{Journals}

Édition électronique

URL : http://journals.openedition.org/assr/26360

DOI : $10.4000 /$ assr. 26360

ISSN : $1777-5825$

Éditeur

Éditions de l'EHESS

Édition imprimée

Date de publication : 31 décembre 2014

Pagination : 163

ISBN : 978-2-7132-2467-6

ISSN : 0335-5985

\section{Référence électronique}

Michel Ostenc, « Giorgio Chiosso, Libri di scuola e mercato editorial. Dal primo Ottocento alla Riforma Gentile», Archives de sciences sociales des religions [En ligne], 168 | 2014, mis en ligne le 09 avril 2015, consulté le 22 septembre 2020. URL : http://journals.openedition.org/assr/26360 ; DOI : https:// doi.org/10.4000/assr.26360

Ce document a été généré automatiquement le 22 septembre 2020.

(c) Archives de sciences sociales des religions 


\section{Giorgio Chiosso, Libri di scuola e mercato editorial. Dal primo Ottocento alla Riforma Gentile}

Milano, Franco Angeli, 2013, 223 p.

Michel Ostenc

\section{RÉFÉRENCE}

Giorgio Chiosso, Libri di scuola e mercato editorial. Dal primo Ottocento alla Riforma Gentile, Milano, Franco Angeli, 2013, 223 p. 
1 L'orientation cléricale et conservatrice de l'enseignement piémontais au début de la Restauration resta sans lendemain, l'ascension sociale de la bourgeoisie contribuant à diffuser une conception plus libérale de l'alphabétisation du peuple. Le maître d'école remplaça progressivement le prêtre dans les esprits. L'ouvrage de Giorgio Chiosso vient combler une lacune dans l'histoire de l'éducation en Italie au XIX ${ }^{\mathrm{e}}$ siècle par une étude précise et documentée de l'édition scolaire dans l'ensemble de la péninsule

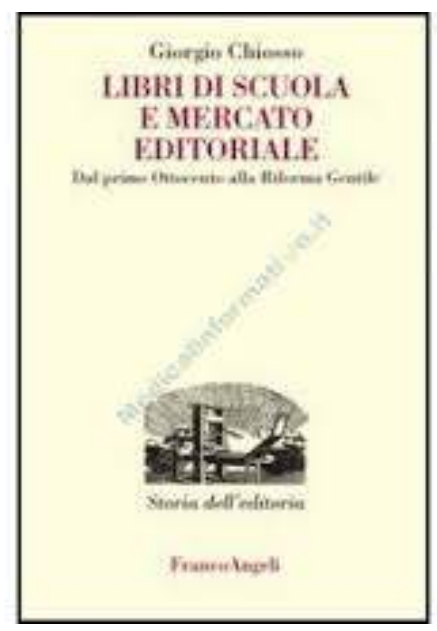
après son unité.

2 L'édition piémontaise de l'époque de la Restauration ouvrait une période de transition entre le livre d'instruction du $\mathrm{XVIII}^{\mathrm{e}}$ siècle et le manuel scolaire. Des réticences se manifestaient pourtant parmi les familles et dans les administrations à l'égard des pratiques didactiques qui utilisaient des abécédaires dans des enseignements collectifs en abandonnant la méthode individuelle et le catéchisme. Au cours des années 1830, la presse piémontaise manifesta un intérêt particulier pour les asiles d'enfants et pour les écoles primaires. Les initiatives philanthropiques et l'action des congrégations religieuses s'étendirent aux enfants pauvres et commencèrent à se préoccuper de la formation des maîtres. Elles répondaient à une demande d'instruction croissante venant de familles d'artisans et d'employés désireuses d'assurer la promotion de leurs enfants. La loi Boncompagni (1848) et les règlements qui en découlèrent eurent d'importantes conséquences. L'édition scolaire devint une affaire rentable, évoluant du livre pour la jeunesse studieuse vers le manuel scolaire. Au début des années 1850, les autorités piémontaises souhaitaient rendre l'enseignement public plus homogène en ayant recours à des livres scolaires approuvés par l'État. L'hégémonie pédagogique des Jésuites dans le secondaire était progressivement remplacée par une idéologie centralisatrice qui devint une caractéristique du "piémontisme scolaire"; mais, à l'aube de l'unité italienne, $40 \%$ des élèves fréquentaient des établissements privés. La plupart d'entre eux suivaient les cours de "séminaires mixtes », n'exigeant aucun engagement à la prêtrise. Ces établissements étaient particulièrement nombreux dans les États de l'Église (Marches, Romagne et Ombrie) et dans le royaume des Deux-Siciles. Les enfants entraient au séminaire à $10 \mathrm{ans}$, âge trop tendre pour une vocation religieuse, et ils recevaient un enseignement d'un niveau inférieur à celui des établissements publics. En 1876, le nombre d'élèves des séminaires s'était accru, $20 \%$ d'entre eux seulement se destinant à la prêtrise. Les enseignants des séminaires n'avaient généralement aucune habilitation, sauf au Piémont, en Lombardie et en Vénétie ; mais les droits d'inscription étaient moins élevés qu'au lycée et le séminaire était souvent le seul établissement d'enseignement secondaire dans les régions les moins favorisées. L'enseignement catholique était animé d'une nostalgie du passé et il éprouvait du ressentiment à l'égard des intrusions de l'État. Giorgio Chiosso nous donne des éclaircissements à son sujet. Ses manuels provenaient de l'éditeur Marietti à Turin et de la Tipografia Calsanziana des Frères de la Doctrine chrétienne à Florence. Bien qu'il fût hostile au Risorgimento, Jean Bosco fréquentait les milieux libéraux et sa 
pensée pédagogique, exposée en 1877 dans «Il sistema preventivo nell'educazione della gioventù ", traduisait sa foi dans la jeunesse et son souci d'une formation de bons chrétiens et d'honnêtes citoyens. La Librairie salésienne était liée aux ambitions scolaires de la congrégation. Ses publications de littérature italienne et d'humanités classiques s'adressaient au gymnase plus qu'au lycée. Elles dispensaient une éducation chrétienne distincte de celle de l'intransigeance catholique au lendemain de l'Unité italienne.

3 Un nouveau climat culturel naquit dans les années 1870, avec l'apparition d'une pédagogie laïque importée de France et d'Allemagne. L'édition scolaire restait très morcelée entre des entreprises artisanales, quelques maisons seulement obéissant à une véritable gestion éditoriale. C'était le cas de Paravia et de Loescher à Turin qui multipliaient leurs publications scolaires en s'ouvrant au marché italien tout entier. Le monde académique contribua également à cet essor, avec des anthologies destinées à l'enseignement de l'histoire et de la philosophie, du latin et du grec, de l'algèbre et de la géométrie. Les ouvrages les plus nombreux étaient pourtant destinés à l'école primaire, parfois sous forme de livres récréatifs. Le succès de l'édition scolaire piémontaise venait de sa capacité à produire des ouvrages aux difficultés didactiques graduées. Ils visaient la formation du citoyen à travers des récits et des exemples de moralité. Un tournant important fut pris avec l'apparition de la philologie allemande dans l'enseignement des humanités. Cette orientation nouvelle se heurta à la rhétorique des milieux classiques. La méthode philologique faisait reposer l'étude du grec et du latin sur les textes, alors que la rhétorique insistait sur la forme et la composition. Giorgio Chiosso montre combien s'affrontaient ainsi deux conceptions de l'éducation et de la société moderne. Les rhéteurs classiques considéraient que l'ordre social était étroitement lié à la conception religieuse de la vie. La philologie pensait qu'il découlait de la rigueur scientifique. L'éditeur Loescher profita des programmes de 1887 qui faisaient explicitement référence à la méthode philologique. Il publia la «Grammatica greca» de Georg Curtius qui avait fait date dans l'histoire de la linguistique indoeuropéenne et s'imposa sur le marché des textes grecs.

Paravia apparut également dans les années 1870 pour devenir une véritable maison d'édition. Son succès fut lié à une publicité moderne et à des textes scolaires enrichis d'illustrations, sans augmentation de prix. Paravia publiait des ouvrages relevant de la tradition spiritualiste piémontaise aussi bien que d'une sensibilité positiviste. La notion d'histoire nationale ne fit son apparition que dans les programmes de 1888. Les deux ouvrages essentiels étaient "La Storia d'Italia raccontata ai giovinetti» de Giuseppe La Farina et "La Storia d'Italia raccontata alla gioventù » de Jean Bosco. La «Breve storia d'Europa, specialmente d'Italia » d'Ettore Ricotti, plusieurs fois rééditée chez Paravia, était pleine d'admiration pour la monarchie piémontaise. L'enseignement de l'histoire était conçu «magistra vitae» dans les premières classes primaires et devenait ensuite une introduction à l'épopée nationale du Risorgimento. L'objectif recherché était la valorisation de la patrie italienne.

5 Avant l'unité italienne, les enfants apprenaient à lire dans des livres récréatifs. Le premier prototype de livre de lecture fut le "Giannetto" (1837) de Luigi Parravicini, ouvrage de large diffusion constamment réédité jusqu'en 1910. Cette encyclopédie pleine de bon sens dispensait au peuple une instruction civique, une formation aux bonnes manières et quelques rudiments d'histoire. Par la suite, le livre de lecture occupa une place centrale dans l'enseignement. À l'exception de la Toscane, la langue 
italienne était presque inconnue au moment de l'unité et les italophones largement minoritaires. L'usage de l'italien devint obligatoire et Giorgio Chiosso montre qu'il fut conçu non seulement comme instrument d'unification, mais aussi de rapprochement des classes sociales. L'enseignement de la langue fut donc étroitement associé à la notion de patrie qui précéda en Italie celle de la Nation. La patrie était empreinte de notions émotives qui lui conféraient une filiation avec la famille et le village natal. L'idéal du bon citoyen était étroitement associé à celui du travail et ses devoirs passaient par l'obligation fiscale et par celle du service militaire. Le maître devenait ainsi un bienfaiteur de l'humanité. Cette conception quasi maternelle d'une Italie protectrice de ses habitants permettait de concilier les nécessités nationales avec des sensibilités régionalistes toujours présentes. Les programmes de l'enseignement primaire de Guido Baccelli (1894) faisaient passer l'éducation du peuple bien avant son instruction.

6 Les programmes de 1888 provoquèrent un afflux de livres scolaires qui suscita les critiques des enseignants. Les livres de lecture étaient jugés trop abstraits et nombre d'instituteurs préféraient utiliser en classe des extraits de De Amicis ou de Collodi qui touchaient davantage les enfants. La commission des livres scolaires (1894) présidée par l'inspecteur général Giuseppe Chiarini se montra d'une grande sévérité, exigeant des manuels une exactitude scientifique rigoureuse, une typographie soignée et des illustrations en harmonie avec le texte. Chiarini estimait nécessaire l'étude des langues modernes, des droits et des devoirs du citoyen dans l'enseignement secondaire classique. De nombreux éditeurs très présents dans le secteur scolaire furent contraints comme Bemporad à Florence d'adapter leurs manuels aux exigences des commissions de contrôle. L'audience du livre de lecture de Pietro Dazzi s'étendit aux familles populaires et les manuels de Raffaele Zeno s'inspirèrent de l'observation et de l'expérimentation. Le marché du livre fut dynamisé par la culture positiviste et les éditions Hoepli surent l'occuper grâce aux liens tissés avec le mathématicien Francesco Brioschi de l'École Polytechnique de Milan. Avec l'essor de l'édition milanaise, la principale nouveauté concerna le développement de maisons de province, comme Zanichelli à Bologne et Sandron à Palerme. Le recul de l'analphabétisme de 75 à $50 \%$ de la population entre 1860 et 1900 , et la diffusion de l'enseignement secondaire contribuèrent au succès de l'édition scolaire.

$7 \mathrm{Au}$ début $\mathrm{du} \mathrm{xx}^{\mathrm{e}}$ siècle, le marché de l'édition scolaire était dominé par les grandes maisons du nord et du centre du pays : Vallardi à Milan, Paravia à Turin, Barbera et Bemporad à Florence. La loi Orlando de 1904 leur donna une nouvelle impulsion avec la création d'un enseignement postprimaire. Bemporad demanda aux écrivains Renato Fucini et Matilde Serao d'écrire des manuels destinés à cet enseignement. Il publia le " Libretto verde » d'Alfredo Della Pane qui lui était destiné et multiplia les syllabaires. Les programmes de 1905 faisaient plus de place à la géographie conçue comme instruction civique et patriotique. Les Atlas de Bemporad profitèrent de la présence des sociétés géographiques et photographiques nationales à Florence pour améliorer leurs illustrations. La réforme Gentile de 1923 changea profondément le climat culturel du pays. Elle eut un grand retentissement dans l'édition scolaire, faisant disparaître des entreprises incapables de s'adapter aux nouveaux programmes et en incitant d'autres à se reconvertir dans la publication d'atlas ou de dictionnaires. La commission chargée de la révision des livres scolaires se montra draconienne. Giuseppe Lombardo-Radice qui la présidait concevait le manuel scolaire en fonction des capacités de l'élève. La pédagogie idéaliste percevait l'enfance comme un âge créatif dans une perspective 
héritée du romantisme de Pestalozzi et de Froebel. L'enseignement secondaire dispensait une culture humaniste destinée à renouveler la classe dirigeante du pays. La réforme Gentile provoqua une réorientation de Paravia qui abandonna progressivement le secteur de l'instruction primaire pour se spécialiser dans l'enseignement secondaire et le matériel didactique. L'éditeur turinois publia la «Storia della letteratura italiana " de Domenico Bulferetti et des textes de nouveaux auteurs comme Ciro Trabalza et Ettore Allodoli. Il conçut des cartes murales illustrant les enseignements de géographie, de physique, de chimie et de sciences naturelles. Les auteurs proches de Gentile utilisèrent par contre les éditeurs Sandron, Laterza et Vallecchi, puis La Nuova Italia et Sansoni. L'éditeur milanais Mondadori sut s'assurer le concours d'auteurs de qualité comme Virgilio Brocchi, auteur de l'ouvrage pour enfants «La storia di Allegretto e Serenella», ou Dante Dini (Alla porta del cuore) (Lo Zufolo). Vallecchi pouvait compter sur le soutien du disciple florentin de Gentile, Ernesto Codignola. Les éditeurs Cappelli et Zanichelli (Bologne), Vallardi (Milan) et Vallecchi (Florence) signèrent une pétition de soutien au gouvernement de Mussolini au moment du Manifeste des intellectuels fascistes (1925).

8 Les mérites de l'ouvrage de Giorgio Chiosso ne se limitent pas à une étude des manuels et de l'édition scolaires. Au-delà de ce caractère informatif, l'auteur parvient à immerger sa réflexion dans la vie même de l'école italienne et à en restituer l'esprit. Il contribue ainsi à un apport majeur à l'histoire de l'éducation en Italie. 